\title{
The weak sequential core for two-period economies
}

Citation for published version (APA):

Predtetchinski, A., Herings, P. J. J., \& Perea ý Monsuwé, A. (2002). The weak sequential core for twoperiod economies. METEOR, Maastricht University School of Business and Economics. METEOR Research Memorandum No. 011 https://doi.org/10.26481/umamet.2002011

Document status and date:

Published: 01/01/2002

DOI:

10.26481/umamet.2002011

Document Version:

Publisher's PDF, also known as Version of record

\section{Please check the document version of this publication:}

- A submitted manuscript is the version of the article upon submission and before peer-review. There can be important differences between the submitted version and the official published version of record.

People interested in the research are advised to contact the author for the final version of the publication, or visit the DOI to the publisher's website.

- The final author version and the galley proof are versions of the publication after peer review.

- The final published version features the final layout of the paper including the volume, issue and page numbers.

Link to publication

\footnotetext{
General rights rights.

- You may freely distribute the URL identifying the publication in the public portal. please follow below link for the End User Agreement:

www.umlib.nl/taverne-license

Take down policy

If you believe that this document breaches copyright please contact us at:

repository@maastrichtuniversity.nl

providing details and we will investigate your claim.
}

Copyright and moral rights for the publications made accessible in the public portal are retained by the authors and/or other copyright owners and it is a condition of accessing publications that users recognise and abide by the legal requirements associated with these

- Users may download and print one copy of any publication from the public portal for the purpose of private study or research.

- You may not further distribute the material or use it for any profit-making activity or commercial gain

If the publication is distributed under the terms of Article $25 \mathrm{fa}$ of the Dutch Copyright Act, indicated by the "Taverne" license above, 


\title{
The Weak Sequential Core for Two-period Economies*
}

\author{
Arkadi Predtetchinski $^{\dagger} \quad$ P. Jean-Jacques Herings ${ }^{\dagger}$ \\ Andres Perea
}

March, 2002

\begin{abstract}
We adapt the core concept to deal with economies in which trade in assets takes place at period 1, uncertainty about asset payoffs is released at period 2, and agents trade in commodities afterwards. We define the weak sequential core as the set of allocations that are stable against coalitional deviations ex ante, and moreover cannot be improved upon by any coalition once the uncertainty is being released. We restrict ourselves to credible deviations, i.e. coalitional deviations at period 1 that cannot be counterblocked by some subcoalition at period 2. We study the relationship of the resulting core concept with other sequential core concepts, give sufficient conditions under which the weak sequential core is non-empty, but show that it is possible to give reasonable examples where it is empty.
\end{abstract}

JEL-classification: C71, C73, D52

Keywords: Core, assets.

*Helpful comments of Hans Peters are gratefully acknowledged.

${ }^{\dagger}$ Department of Economics, Universiteit Maastricht, P.O. Box 616, 6200 MD Maastricht, The Netherlands. Tel.: +31-43-38883635, fax: +31-43-38884878, e-mail: a.predtet chinski@algec . unimaas.nl, p.herings@algec . unimaas.nl.

$\ddagger$ Department of Quantitative Economics, Universiteit Maastricht, P.O. Box 616, 6200 MD Maastricht, The Netherlands. Tel.: +31-43-3883288, fax: +31-43-3884874, e-mail: a.perea@ke.unimaas.nl. 


\section{Introduction}

One of the central questions in the theory on resource allocation is which distributions are to be regarded as self-enforcing, or stable. There seems to exist a large consensus on a necessary condition for stable allocations, namely that no coalition should be able to improve upon it by gathering and redistributing its resources among the coalition members, without using the resources of others. The latter property is referred to as the core condition. While this property may have an unambiguous meaning in purely static contexts, it is not obvious, a-priori, how to formalize the principle in situations where there is initial uncertainty about some parameters of the economy, to be released at later stages. The reason is that a coalition, which ex-ante has no incentive to block a given allocation since it cannot improve upon it in expected terms, may object against it once the state of nature is revealed. A similar argument may be made when the distribution of resources takes place at various points in time, instead of once at the initial stage.

It thus seems necessary to refine the "classical" core concept for the above mentioned situations, requiring an allocation not only to be stable at the beginning, but in addition to remain stable against possible deviations once the uncertainty is released, or once the dynamic cooperation process is under way. Such sequential core concepts have been investigated by Gale (1978), Becker and Chakrabarti (1995), Repullo (1988), and Koutsougeras (1998) for specific economic environments, whereas Kranich, Perea and Peters (2001) propose two core concepts for situations where agents face a finite sequence of transferable utility games: the strong sequential core and the weak sequential core.

Predtetchinski, Herings and Peters (2001) apply an appropriately adapted version of the strong sequential core to two-stage economies in which trade in assets takes place at period zero and trade in commodities at period one. The payoff to each asset is uncertain at period zero, but is revealed before trade in commodities takes place. The idea in the strong sequential core concept is to select those state contingent allocations which are stable against coalitional deviations ex-ante, and moreover cannot be improved upon by any coalition once the uncertainty is being released. It may be the case, however, that the deviation by a coalition that promised improvement in the ex ante terms, can in turn be improved upon by some sub-coalition once the true state of nature is known. In this case, it could be argued that the 
coalitional deviation at hand is not "credible" since it may be counterblocked by some subcoalition. By imposing the weaker restriction that state contingent allocations should be robust solely against "credible" deviations, the concept of the weak sequential core is obtained, which is the subject of study in the present paper.

For static cooperative situations, it is known that the restriction to credible deviations by coalitions does not alter the definition of the core: an allocation can be blocked by some coalitional deviation if and only if it can be blocked by a credible coalitional deviation (see Ray, 1989). Kranich, Perea and Peters (2001) show, however, that this restriction becomes relevant if one turns to dynamic cooperative environments. As will become clear in this paper, the focus on credible deviations is crucial as well for our context of two-stage economies. Consequently, the weak sequential core for such economies is in general a strict superset of the strong sequential core.

The concept of the Two-Stage core, due to Koutsougeras (1998), is obtained under the assumption that if a deviation occurs before the true state of nature is known, no exchange can take place within the deviating coalition once the state has been revealed. Whenever some weak conditions are satisfied, the weak sequential core is a subset of the two-stage core.

In Predtetchinski, Herings and Peters (2001), it is shown that the strong sequential core is empty for a large family of economies. In fact, generic emptiness is shown in the setting of a finance economy, when asset markets are not complete. Moreover, an example of the economy with multiple consumption goods is reported, where an asset structure satisfies a very strong condition of completeness, and yet the strong sequential core is empty.

In contrast, the weak sequential core has been shown to be nonempty in a number of important special cases. One instance is the finance economy, where the weak sequential core coincides with the two-stage core. Whenever a complete set of state-contingent contracts is available for trade, co-existence of the weak sequential core, strong sequential core, and the two-stage core holds. Finally, we prove that the weak sequential core is non-empty for economies with two agents.

Surprisingly, we give an example with three agents and no assets where the weak sequential core fails to exist. For the case of the strong sequential core, it was easily seen that it is weakly increasing in the number of assets. This property no longer applies, though, for the weak sequential core. In fact, we demonstrate that the weak 
sequential core may be non-empty in an economy with no assets, while becoming empty after introducing a single asset in this economy.

The outline of the paper is as follows. In Section 2 the model is described. In Section 3 the concept of the weak sequential core is introduced and is compared to the concepts of the strong sequential core and the two-stage core. Some special cases are considered in Section 4. Section 5 introduces an NTU-game associated with the two-period economy. Section 6 contains examples.

\section{The Model}

We consider an economy with two time periods $(t=0, t=1)$ and uncertainty concerning the state of nature in period 1. We assume that for each state $s$ in the set $S=\{1, \ldots, S\}$ probability $\rho_{s}>0$ of occurrence is objectively known.

There is a set $N=\{1, \ldots, n\}$ of agents. Agents trade in $J$ assets in period 0 and, conditional on the realization of the state of nature $s$, in $L$ commodities in period 1 . In state of nature $s \in S$, agent $i$ has a consumption set $X_{s}^{i} \subset \mathbb{R}^{L}$. The ex-ante consumption set of agent $i$ is given by $X^{i}=\prod_{s \in S} X_{s}^{i}$. We write $X=\prod_{i \in N} X^{i}$. An agent $i$ is further characterized by his vector of initial endowments in state of nature $s$, $\omega_{s}^{i} \in X_{s}^{i}$, and his elementary (Bernoulli) utility function $u_{s}^{i}: X_{s}^{i} \mapsto \mathbb{R}$. Agents are expected utility maximizers, with $v^{i}: X^{i} \mapsto \mathbb{R}$ the expected utility function defined by $v^{i}\left(x^{i}\right)=\sum_{s \in S} \rho_{s} u_{s}^{i}\left(x_{s}^{i}\right)$.

The matrix of asset payoffs is given by the $S L \times J$ matrix $A$. Generic entry $A_{s, l}^{j}$ of the matrix $A$ specifies the quantity of commodity $l$ paid by asset $j$ in state of nature $s$.

These components together define our economy, denoted by $\mathcal{E}$. The institutional set-up of the economy is as follows.

1. In period 0, trade in assets takes place. Alternatively, one may think of these trades taking the form of state-contingent contracts. There is no endowment and therefore no consumption in period $t=0$.

2. Nature randomly chooses the state of nature. The execution of asset contracts takes place and results in an allocation $x$.

3. In period 1, trade in commodities takes place. Agents treat allocation $x$ as their initial endowments. Trade in commodities results in an allocation $y$ of commodities, which is consumed. 
Notice that the institutional setting is one of dynamic exchange without the formation of prices. Our analysis is therefore complementary to the extensive literature on constrained suboptimality of competitive equilibria when asset markets are incomplete (see for instance Geanakoplos and Polemarchakis, 1986).

An important role in our analysis is played by the sets

$$
\begin{aligned}
\mathcal{A}(M)=\left\{x \in \prod_{i \in M} X^{i} \mid \quad\right. & \sum_{i \in M} x^{i}=\sum_{i \in M} \omega^{i}, \\
& \left.x^{i}-\omega^{i} \in\langle A\rangle, \quad \forall i \in M\right\},
\end{aligned}
$$

where $M \subseteq N$ is a coalition of agents, and $\langle A\rangle$ denotes the linear space spanned by the columns of $A$, i.e., the span of $A$. Allocations in the set $\mathcal{A}(M)$ are regarded as $A$-feasible for the coalition $M$. These are those allocations that can be achieved by trade in assets in period 0 within the coalition $M$. If there are no assets, then we define $\mathcal{A}(M)=$ $\left\{\left(\omega^{i}\right)_{i \in M}\right\}$.

Any $A$-feasible allocation $x \in \mathcal{A}(M)$ gives rise to exactly $S$ ex post sub-economies $\mathcal{E}_{s}(x, M)$, one in each of the possible states of nature $s \in S$, following the resolution of uncertainty. The sub-economy associated with the state of nature $s$ has a set of agents $M$, commodity space $\mathbb{R}^{L}$, consumption sets $X_{s}^{i}$, utility functions $u_{s}^{i}$, and initial endowments $x_{s}^{i}$. Formally,

$$
\mathcal{E}_{s}(x, M)=\mathcal{E}\left(M, \mathbb{R}^{L},\left\langle X_{s}^{i}, u_{s}^{i}, x_{s}^{i}\right\rangle_{i \in M}\right) .
$$

The classical core of the economy $\mathcal{E}_{s}(x, M)$ is denoted by $C\left(\mathcal{E}_{s}(x, M)\right)$, hence

$$
\begin{aligned}
& C\left(\mathcal{E}_{s}(x, M)\right)=\left\{y_{s} \in \prod_{i \in M} X_{s}^{i} \mid \sum_{i \in M} y_{s}^{i}=\sum_{i \in M} x_{s}^{i}\right. \\
& \text { there are no } Q \subseteq M \text { and } \widetilde{y}_{s} \in \prod_{i \in Q} X_{s}^{i} \text { such that } \\
& \left.\sum_{i \in Q} \widetilde{y}_{s}^{i}=\sum_{i \in Q} x_{s}^{i} \text { and } u_{s}^{i}\left(y_{s}^{i}\right)<u_{s}^{i}\left(\widetilde{y}_{s}^{i}\right) \text { for all } i \in Q\right\}
\end{aligned}
$$

for all $s \in S, M \subseteq N$, and $x \in \mathcal{A}(M)$.

\section{Three concepts of the sequential core}

In this section we compare the concept of the weak sequential core to that of the strong sequential core and of the two-stage core. We start with the definition of the weak sequential core.

Definition 1 An allocation $\bar{y}$ is an element of the weak sequential core of the economy $\mathcal{E}$, denoted $W S C(\mathcal{E})$, if 
1. there exists an $\bar{x} \in \mathcal{A}(N)$ such that $\bar{y}_{s} \in C\left(\mathcal{E}_{s}(\bar{x}, N)\right)$ for all $s \in S$,

2. there do not exist $M \subseteq N, y \in \prod_{i \in M} X^{i}$, and $x \in \mathcal{A}(M)$ such that

$y_{s} \in C\left(\mathcal{E}_{s}(x, M)\right)$ for all $s \in S$

$v^{i}\left(y^{i}\right)>v^{i}\left(\bar{y}^{i}\right)$ for all $i \in M$.

Part one of the definition is the requirement that the allocation $\bar{y}$ be robust against the deviations that can potentially take place at period one, after the true state of nature has been revealed. Part two of the definition is the requirement that the allocation $\bar{y}$ be robust against all credible deviations at period zero, before the true state of nature is known.

The three concepts of the sequential core - weak sequential core, strong sequential core and two-stage core - agree on the meaning of the improvement by a coalition at period one. Since agents face no uncertainty and no prospects of future consumption at period one, the classical definition of an improving coalition seems to be appropriate, with the initial positions of coalitions being determined by the allocation $\bar{x}$. This results in the requirement that $\bar{y}_{s}$ be in the classical core of the economy $\mathcal{E}_{s}(\bar{x}, N)$, for all the states $s \in S$.

The situation differs as we turn to period zero. It is not obvious which deviations should be taken into account, and which not. Whence, a discrepancy between the three concepts of the sequential core as to the definition of an improvement by a coalition for period zero. The idea in the weak sequential core is to focus on credible deviations.

Suppose that a coalition $M$ considers to deviate at period zero to allocation $y$, which is preferred, in expected terms, to allocation $\bar{y}$ by all the members of $M$. In general, there may be counter-deviations from $y$ by sub-coalitions of $M$ once the true state of nature is known. Coalition $M$, however, might redistribute assets among its members at period zero in such a way that, given this redistribution, no deviations from $y$ will be profitable in the subsequent period. If there does exist such a redistribution of assets within the coalition $M$, then $y$ can be regarded as a credible deviation. Otherwise it is not credible, for there may be counter-deviations from $y$ at period one. The weak sequential core requires its elements to be robust only against credible deviations, rather than against all possible deviations in the classical sense. 
Below we reproduce the definition of the strong sequential core from Predtetchinski, Herings, and Peters (2001).

Definition 2 An allocation $\bar{y}$ is an element of the strong sequential core of the economy $\mathcal{E}$, denoted $S S C(\mathcal{E})$, if

1. there exists an $\bar{x} \in \mathcal{A}(N)$ such that $\bar{y}_{s} \in C\left(\mathcal{E}_{s}(\bar{x}, N)\right)$ for all $s \in S$,

2. there do not exist $M \subseteq N$ and $y \in \prod_{i \in M} X^{i}$ such that

$\sum_{i \in M} y^{i}=\sum_{i \in M} \omega^{i}$

$v^{i}\left(y^{i}\right)>v^{i}\left(\bar{y}^{i}\right)$ for all $i \in M$.

The difference between Definition 1 and Definition 2 is that the latter does not require deviations of coalitions at period zero to be robust to possible counter-deviations by sub-coalitions in period one. Instead, the classical notion of improvement is adopted: a coalition improves upon the allocation $\bar{y}$ by the allocation $y$, if the allocation $y$ is feasible and gives each member of the deviating coalition more expected utility than the allocation $\bar{y}$ does. Any element of the strong sequential core must therefore be stable against all the deviations in the classical sense, whether credible or not. Thus, the strong sequential core is a subset of the weak sequential core.

We proceed with the definition of the two-stage core, due to Koutsougeras (1998).

Definition 3 An allocation $\bar{y}$ is an element of the two-stage core of the economy $\mathcal{E}$, denoted $T S C(\mathcal{E})$, if

1. there exists an $\bar{x} \in \mathcal{A}(N)$ such that $\bar{y}_{s} \in C\left(\mathcal{E}_{s}(\bar{x}, N)\right.$ ) for all $s \in S$,

2. there do not exist $M \subseteq N$ and $x \in \mathcal{A}(M)$ such that $v^{i}\left(x^{i}\right)>$ $v^{i}\left(\bar{y}^{i}\right)$ for all $i \in M$.

Unlike Definitions 1 and 2, Definition 3 assumes that a coalition can only use $A$-feasible allocations to improve at period zero: no exchange can take place within the deviating coalition after the uncertainty has been resolved. The coalition that deviates at period zero is thus confined in its choice of the consumption bundles to those that can be allocated by means of exchange of assets alone.

The relationship between the three concepts of the sequential core is summarized by the following theorem. 


\section{Theorem 1}

1. $S S C(\mathcal{E}) \subseteq W S C(\mathcal{E})$,

2. Suppose that $X_{s}^{i}$ is closed, convex and bounded from below, $u_{s}^{i}$ is continuous and quasi-concave for all $i \in N, s \in S$. Then $W S C(\mathcal{E}) \subseteq T S C(\mathcal{E})$.

In fact, the inclusion of the weak sequential core in the two-stage core holds, whenever the classical cores of the economies $\mathcal{E}_{s}(x, M)$ are non-empty for all $s \in S, M \subseteq N$, and $x \in \mathcal{A}(M)$. This is guaranteed by the conditions of the second part of Theorem 1. Otherwise there may be less possibilities for the deviating coalitions to form within the weak sequential core than within the two-stage core. In this case the weak sequential core need not be a subset of the two-stage core.

Proof. The first part of Theorem 1 is obvious. To prove the second part, consider an allocation $\bar{y} \in W S C(\mathcal{E})$ and suppose that $\bar{y} \notin T S C(\mathcal{E})$. Then there exists a coalition $M \subseteq N$ and an allocation $x \in \mathcal{A}(M)$ such that

$$
v^{i}\left(x^{i}\right)>v^{i}\left(\bar{y}^{i}\right)
$$

for all $i \in M$. The assumptions of Theorem 1 guarantee that the cores of the economies $\mathcal{E}_{s}(x, M)$ are non-empty for all $s \in S$. Choose allocations $y_{s} \in C\left(\mathcal{E}_{s}(x, M)\right)$, arbitrarily. Then,

$$
v^{i}\left(y^{i}\right) \geq v^{i}\left(x^{i}\right)>v^{i}\left(\bar{y}^{i}\right)
$$

for all $i \in M$. This implies, however, that $\bar{y}$ is not an element of the weak sequential core, a contradiction. We conclude that $\bar{y} \in T S C(\mathcal{E})$.

\section{Some special cases}

In this section we consider two special cases: the case where a complete set of state-contingent contracts is available, and the case of a finance economy.

We start with the case where for each commodity $l$ and each state of nature $s$ there is a contract specifying the delivery of commodity $l$ contingent on the occurrence of state of nature $s$. The total number of 
state-contingent contracts is therefore $S L$. When all of these statecontingent contracts are available, $A$ is the identity matrix. More generally, we can require the span of $A$ to be the whole space $\mathbb{R}^{S L}$. If this is indeed the case, then the weak sequential core, the strong sequential core, and the two-stage core coincide.

Theorem 2 If $\langle A\rangle=\mathbb{R}^{S L}$, then $S S C(\mathcal{E})=W S C(\mathcal{E})=T S C(\mathcal{E})$.

Next we examine the case of a finance economy. A finance economy is a special case of a two-period economy, where a unique commodity is available in each state of nature, i.e. $L=1$. If $X_{s}^{i}=\mathbb{R}_{+}$and the utility functions $u_{s}^{i}$ are all strongly monotone, then for any state $s \in S$, coalition $M \subseteq N$ and $A$-feasible allocation $x \in \mathcal{A}(M)$,

$$
C\left(\mathcal{E}_{s}(x, M)\right)=\left\{x_{s}\right\} .
$$

This observation leads to the following theorem.

Theorem 3 If $L=1, X_{s}^{i}=\mathbb{R}_{+}$, and $u_{s}^{i}$ is strongly monotone for all $i \in N, s \in S$, then $W S C(\mathcal{E})=T S C(\mathcal{E})$.

The result of Theorem 3 is in sharp contrast to the findings with regard to the strong sequential core. It has been shown in Predtetchinski, Herings and Peters (2001) that, if $J<S-1$, then the strong sequential core of a finance economy is generically empty, and, if $J=S-1$, then, generically, it consists of no more than a finite number of elements. This shows that the weak sequential core may be a strict superset of the strong sequential core.

To conclude the present section we formulate an existence result for the weak sequential core in case of an economy with two agents.

Theorem 4 Suppose that the economy $\mathcal{E}$ satisfies the following assumptions: $n=2, X_{s}^{i}$ are bounded from below, $u_{s}^{i}$ are continuous, and for every $\bar{y}_{s}^{i} \in X_{s}^{i}$ the closure of the set $\left\{y_{s}^{i} \in X_{s}^{i} \mid u_{s}^{i}\left(\bar{y}_{s}^{i}\right) \leq u_{s}^{i}\left(y_{s}^{i}\right)\right\}$ is contained in $X_{s}^{i}$. Then $W S C(\mathcal{E}) \neq \emptyset$.

Proof. First we introduce some additional notation:

$$
\begin{aligned}
& X_{s}(N)=\left\{y_{s} \in \prod_{i \in N} X_{s}^{i} \mid \sum_{i \in N} y_{s}^{i}=\sum_{i \in N} \omega_{s}^{i}\right\} \\
& X(N)=\prod_{s \in S} X_{s}(N) \\
& Z(N)=\left\{(x, y) \in \mathcal{A}(N) \times X(N) \mid u_{s}^{i}\left(x_{s}^{i}\right) \leq u_{s}^{i}\left(y_{s}^{i}\right), \quad s \in S, \quad i \in N\right. \\
&\left.v^{i}\left(\omega^{i}\right) \leq v^{i}\left(y^{i}\right), \quad i \in N\right\} .
\end{aligned}
$$


Observe that $(\omega, \omega) \in Z(N)$. Moreover, under the assumptions of Theorem $4, Z(N)$ is a compact set. Hence, there exists an element $(\bar{x}, \bar{y}) \in Z(N)$ that maximizes the function $\sum_{i \in N} \alpha^{i} v^{i}\left(y^{i}\right)$ over all $(x, y) \in Z(N) ; \alpha^{1}$ and $\alpha^{2}$ are non-negative weights, at least one of which is positive. In the remainder of the proof we show that the allocation $\bar{y}$ is an element of the weak sequential core.

Notice that $(\bar{x}, \bar{y})$ satisfies the following inequalities:

$$
\begin{aligned}
u_{s}^{i}\left(\bar{x}_{s}^{i}\right) \leq u_{s}^{i}\left(\bar{y}_{s}^{i}\right) \\
v^{i}\left(\omega^{i}\right) \leq v^{i}\left(\bar{y}^{i}\right) .
\end{aligned}
$$

Step 1.

$$
\begin{aligned}
& (\bar{x}, \bar{y}) \text { satisfies the first part of Definition } 1 \text { : } \\
& \bar{y}_{s} \in C\left(\mathcal{E}_{s}(\bar{x}, N)\right), \quad s \in S .
\end{aligned}
$$

The inequalities (1) imply that neither agent 1 nor agent 2 is able to improve upon allocation $\bar{y}$ at period 1 given the allocation $\bar{x}$. Suppose that the grand coalition is able to improve upon $\bar{y}$ at some state $s_{0} \in$ $\{1, \ldots S\}$. Then there is $y_{s_{0}} \in X_{s_{0}}(N)$ such that

$$
u_{s_{0}}^{i}\left(y_{s_{0}}^{i}\right)>u_{s_{0}}^{i}\left(\bar{y}_{s_{0}}^{i}\right), i \in N
$$

Define the allocation $\tilde{y}$ as follows:

$$
\tilde{y}_{s}^{i}= \begin{cases}y_{s_{0}}^{i}, & \text { if } s=s_{0} \\ \bar{y}_{s}^{i}, & \text { otherwise }\end{cases}
$$

for all $i \in N$. Then $\tilde{y} \in X(N)$ and, moreover,

$$
u_{s}^{i}\left(\tilde{y}_{s}^{i}\right) \geq u_{s}^{i}\left(\bar{y}_{s}^{i}\right)
$$

for all $i \in N, s \in S$ with a strict inequality corresponding to state $s=s_{0}$. It follows from (3) that

$$
v^{i}\left(\tilde{y}^{i}\right)>v^{i}\left(\bar{y}^{i}\right)
$$

for all $i \in N$. Inequalities (3)-(4), together with (1)-(2) also imply that the allocation $(\bar{x}, \tilde{y})$ is an element of $Z(N)$ and that

$$
\sum_{i \in N} \alpha^{i} v^{i}\left(\tilde{y}^{i}\right)>\sum_{i \in N} \alpha^{i} v^{i}\left(\bar{y}^{i}\right)
$$


This contradicts the choice of $(\bar{x}, \bar{y})$.

Step 2. $(\bar{x}, \bar{y})$ satisfies the second part of Definition 1.

Inequalities (2) imply that neither agent 1 nor agent 2 can improve upon $\bar{y}$ at period zero. Suppose that the grand coalition is able to improve upon $\bar{y}$ at period zero. Then there exists $(x, y) \in \mathcal{A}(N) \times$ $X(N)$ such that

$$
\begin{gathered}
y_{s} \in C\left(\mathcal{E}_{s}(x, N)\right), \quad \forall s \in S \\
v^{i}\left(y^{i}\right)>v^{i}\left(\bar{y}^{i}\right), \quad \forall i \in N .
\end{gathered}
$$

It follows from (5) that $u_{s}^{i}\left(x_{s}^{i}\right) \leq u_{s}^{i}\left(y_{s}^{i}\right)$, and from (6) and (2) that $v^{i}\left(\omega^{i}\right)<v^{i}\left(y^{i}\right)$. Therefore, $(x, y)$ is an element of $Z(N)$, and

$$
\sum_{i \in N} \alpha^{i} v^{i}\left(y^{i}\right)>\sum_{i \in N} \alpha^{i} v^{i}\left(\bar{y}^{i}\right)
$$

This contradicts the choice of $(\bar{x}, \bar{y})$.

\section{An NTU-game for the two-period economy}

It is well known that, given a static economy, one can define an associated NTU-game in such a way that the core of the underlying economy is non-empty if and only if the core of the derived NTU-game is non-empty. Below we provide a similar criterion for non-emptiness of the weak sequential core based on an NTU-game associated with the two-period economy. This criterion for non-emptiness will be helpful in the next subsection.

For a given coalition $M \subseteq N, A$-feasible allocation $x \in \mathcal{A}(M)$ and state $s \in S$ let $\left(M, U_{s, x}\right)$ denote the NTU-game associated with the economy $\mathcal{E}_{s}(x, M)$. That is

$$
\begin{aligned}
U_{s, x}(Q)=\left\{u_{s} \in \mathbb{R}^{\{Q\}} \mid \exists y_{s} \in \prod_{i \in Q} X_{s}^{i}: \quad\right. & \sum_{i \in Q} y_{s}^{i}=\sum_{i \in Q} x_{s}^{i}, \\
& \left.u_{s}^{i} \leq u_{s}^{i}\left(y_{s}^{i}\right), i \in Q\right\},
\end{aligned}
$$

for all $Q \subseteq M$. Let $C\left(M, U_{s, x}\right)$ be the core of $\left(M, U_{s, x}\right)$ :

$$
\begin{array}{r}
C\left(M, U_{s, x}\right)=\left\{u \in U_{s, x}(M) \mid \quad \text { there are no } Q \subseteq M \text { and } \widetilde{u} \in U_{s, x}(Q)\right. \\
\text { such that } \left.u^{i}<\widetilde{u}^{i} \text { for all } i \in Q\right\} .
\end{array}
$$




\section{Lemma 1}

1. Let $\bar{y}_{s} \in C\left(\mathcal{E}_{s}(x, M)\right)$. Then $\bar{u}_{s} \in C\left(M, U_{s, x}\right)$, where $\bar{u}_{s}^{i}=u_{s}^{i}\left(\bar{y}_{s}^{i}\right)$ for all $i \in M$.

2. Let $\bar{u}_{s} \in C\left(M, U_{s, x}\right)$. Then there exists $\bar{y}_{s} \in C\left(\mathcal{E}_{s}(x, M)\right)$ such that $\bar{u}_{s}^{i} \leq u_{s}^{i}\left(\bar{y}_{s}^{i}\right)$ for all $i \in M$.

Observe that no assumptions whatsoever are made with respect to the utility functions in Lemma 1, and so the inequality sign in the second part of the lemma cannot in general be replaced with the equality sign. Assuming continuity and strong monotonicity of the functions $u_{s}^{i}$ and setting $X_{s}^{i}=\mathbb{R}_{+}^{L}$, one can argue indeed that for every $\bar{u}_{s}$ in $C\left(M, U_{s, x}\right)$ there exists $\bar{y}_{s}$ in $C\left(\mathcal{E}_{s}(x, M)\right)$ such that $u_{s}^{i}\left(\bar{y}_{s}^{i}\right)$ is exactly equal to $\bar{u}_{s}^{i}$ for all $i \in M$.

Define the NTU-game $(N, V)$ as follows:

$$
\begin{aligned}
V(M) & =\bigcup_{x \in \mathcal{A}(M)} V_{x}(M), \text { where } \\
V_{x}(M) & =\sum_{s \in S} \rho_{s}\left(C\left(M, U_{s, x}\right)-\mathbb{R}_{+}^{M}\right)
\end{aligned}
$$

for all $M \subseteq N$. Let $C(N, V)$ be the core of $(N, V)$.

\section{Theorem 5}

1. Let $\bar{y} \in W S C(\mathcal{E})$. Then $\bar{v} \in C(N, V)$, where $\bar{v}^{i}=v^{i}\left(\bar{y}^{i}\right)$ for all $i \in N$.

2. Let $\bar{v} \in C(N, V)$. Then there exists $\bar{y} \in W S C(\mathcal{E})$ such that $\bar{v}^{i} \leq v^{i}\left(\bar{y}^{i}\right)$ for all $i \in N$.

\section{Proof.}

1. Let $\bar{y} \in W S C(\mathcal{E})$. Then there exists an $\bar{x} \in \mathcal{A}(N)$ such that $\bar{y}_{s} \in C\left(\mathcal{E}_{s}(\bar{x}, N)\right)$ for all $s \in S$. By the first part of Lemma $1, \bar{u}_{s} \in$ $C\left(N, U_{s, \bar{x}}\right)$, where $\bar{u}_{s}^{i}=u_{s}^{i}\left(\bar{y}_{s}^{i}\right)$ for all $i \in N$ and $s \in S$. Therefore, $\bar{v} \in V_{\bar{x}}(N) \subseteq V(N)$, where $\bar{v}=\sum_{s \in S} \rho_{s} \bar{u}_{s}$, and so $\bar{v}^{i}=v^{i}\left(\bar{y}^{i}\right)$ for all $i \in N$.

Suppose that there exist $M \subseteq N$ and $v \in V(M)$ with $\bar{v}^{i}<v^{i}$ for all $i \in M$. Then there exist $x \in \mathcal{A}(M)$ and $u_{s} \in C\left(M, U_{s, x}\right)$ for all $s \in S$ such that $v^{i} \leq \sum_{s \in S} \rho_{s} u_{s}^{i}$ for all $i \in M$. By the second part of Lemma 1, there exist $y_{s} \in C\left(\mathcal{E}_{s}(x, M)\right)$ such that $u_{s}^{i} \leq u_{s}^{i}\left(y_{s}^{i}\right)$ for all $s \in S$ and $i \in M$. Hence, $v^{i}\left(\bar{y}^{i}\right)<v^{i}\left(y^{i}\right)$ for all $i \in M$. This implies, 
however, that $\bar{y} \notin W S C(\mathcal{E})$, a contradiction. We conclude therefore that $\bar{v} \in C(N, V)$.

2. Let $\bar{v} \in C(N, V)$. Then there exist $\bar{x} \in \mathcal{A}(N)$ and $\bar{u}_{s} \in$ $C\left(N, U_{s, \bar{x}}\right)$ for all $s \in S$ such that $\bar{v}^{i} \leq \sum_{s \in S} \rho_{s} \bar{u}_{s}^{i}$ for all $i \in N$. By the second part of Lemma 1, there exist $\bar{y}_{s} \in C\left(\mathcal{E}_{s}(\bar{x}, N)\right)$ such that $\bar{u}_{s}^{i} \leq u_{s}^{i}\left(\bar{y}_{s}^{i}\right)$ for all $s \in S$ and $i \in N$. So, $\bar{v}^{i} \leq v^{i}\left(\bar{y}^{i}\right)$ for all $i \in N$.

Suppose that there exist $M \subseteq N, x \in \mathcal{A}(M)$, and $y_{s} \in C\left(\mathcal{E}_{s}(x, M)\right)$ for all $s \in S$ such that $v^{i}\left(\bar{y}^{i}\right)<v^{i}\left(y^{i}\right)$ for all $i \in M$. Then, by the first part of Lemma $1, u_{s} \in C\left(M, U_{s, x}\right)$ for all $s \in S$, where $u_{s}^{i}=u_{s}^{i}\left(y_{s}^{i}\right)$ for all $i \in M$. Therefore, $v \in V_{x}(M) \subseteq V(M)$, where $v=\sum_{s \in S} \rho_{s} u_{s}$, and so $v^{i}=v^{i}\left(y^{i}\right)$ for all $i \in M$. It follows that $\bar{v}^{i}<v^{i}$ for all $i \in M$. So, $\bar{v} \notin C(N, V)$, a contradiction. We conclude therefore that $\bar{y} \in W S C(\mathcal{E})$.

Consider an economy $\mathcal{E}$ in which all the agents have identical consumption sets and identical state-independent Bernoulli utility functions given by

$$
\begin{aligned}
& X_{s}^{i}=\mathbb{R}_{++}^{L}, \\
& u_{s}^{i}\left(y_{s}^{i}\right)=\frac{1}{L} \sum_{l=1}^{L} \ln \left(y_{s l}^{i}\right), \quad y_{s}^{i} \in X_{s}^{i}
\end{aligned}
$$

for all $i \in N$ and $s \in S$. In the remainder of this section we derive the core of the game $\left(M, U_{s, x}\right)$ for this special case. To do so an additional lemma will be needed.

Let $M \subseteq N, Q \subseteq M, x \in \mathcal{A}(M)$, and $s \in S$ be given. Denote the set of weakly-efficient allocations of the economy $\mathcal{E}_{s}(x, Q)$ by $P\left(\mathcal{E}_{s}(x, Q)\right)$. Hence

$$
\begin{aligned}
& P\left(\mathcal{E}_{s}(x, Q)\right)=\left\{y_{s} \in \prod_{i \in Q} X_{s}^{i} \mid \sum_{i \in Q} y_{s}^{i}=\sum_{i \in Q} x_{s}^{i}\right. \\
& \text { there is no } \widetilde{y}_{s} \in \prod_{i \in Q} X_{s}^{i} \text { such that } \\
& \left.\sum_{i \in Q} \widetilde{y}_{s}^{i}=\sum_{i \in Q} x_{s}^{i} \text { and } u_{s}^{i}\left(y_{s}^{i}\right)<u_{s}^{i}\left(\widetilde{y}_{s}^{i}\right) \text { for all } i \in Q\right\} \text {. }
\end{aligned}
$$

Lemma 2 Suppose that for every $i \in Q$ the set $X_{s}^{i}$ is bounded from below, the function $u_{s}^{i}$ is continuous, and for all $\bar{y}_{s}^{i} \in X_{s}^{i}$ the closure of the set $\left\{y_{s}^{i} \in X_{s}^{i} \mid u_{s}^{i}\left(\bar{y}_{s}^{i}\right) \leq u_{s}^{i}\left(y_{s}^{i}\right)\right\}$ is contained in $X_{s}^{i}$. Then

$$
U_{s, x}(Q)=\left\{u_{s} \in \mathbb{R}^{Q} \mid \exists y_{s} \in P\left(\mathcal{E}_{s}(x, Q)\right): u_{s}^{i} \leq u_{s}^{i}\left(y_{s}^{i}\right), i \in Q\right\} .
$$


Proof. Let $u_{s} \in U_{s, x}(Q)$ and let $\bar{y}_{s} \in \prod_{i \in Q} X_{s}^{i}$ be such that $\sum_{i \in Q} \bar{y}_{s}^{i}=$ $\sum_{i \in Q} x_{s}^{i}$ and $u_{s}^{i} \leq u_{s}^{i}\left(\bar{y}_{s}^{i}\right)$ for all $i \in Q$. Under the assumptions of Lemma 2 there exists a solution $\hat{y}_{s}$ to the following optimization problem:

$$
\begin{aligned}
\operatorname{maximize} & \sum_{i \in Q} u_{s}^{i}\left(y_{s}^{i}\right) \\
\text { subject to } & y_{s} \in \prod_{i \in Q} X_{s}^{i} \\
& \sum_{i \in Q} y_{s}^{i}=\sum_{i \in Q} x_{s}^{i} \\
& u_{s}^{i}\left(\bar{y}_{s}^{i}\right) \leq u_{s}^{i}\left(y_{s}^{i}\right) \text { for all } i \in Q .
\end{aligned}
$$

Moreover, $\hat{y}_{s} \in P\left(\mathcal{E}_{s}(x, Q)\right)$ and $u_{s}^{i} \leq u_{s}^{i}\left(\hat{y}_{s}^{i}\right)$ for all $i \in Q$. This proves the inclusion $\subseteq$. The inclusion $\supseteq$ is obvious.

Observe that the consumption sets and the utility functions as defined by (7) and (8) satisfy the requirements of Lemma 2. Moreover, an allocation $y_{s}$ is an element of the set $P\left(\mathcal{E}_{s}(x, Q)\right)$ if and only if there exist real numbers $t_{s}^{i}>0$ for all $i \in Q$ such that $\sum_{i \in Q} t_{s}^{i}=1$ and $y_{s}^{i}=t_{s}^{i} \sum_{j \in Q} x_{s}^{j}$ for all $i \in Q$. Thus the feasibility set $U_{s, x}(Q)$ can be written

$$
\begin{aligned}
& U_{s, x}(Q)=\left\{u_{s} \in \mathbb{R}^{Q} \mid \begin{array}{l}
\exists t_{s}^{i}>0, i \in Q: \quad \sum_{i \in Q} t_{s}^{i}=1 \\
\\
\left.\exp \left(u_{s}^{i}\right) \leq t_{s}^{i} \beta_{s, x}(Q), i \in Q\right\}, \text { where }
\end{array}\right. \\
& \beta_{s, x}(Q)=\left(\prod_{l=1}^{L} \sum_{i \in Q} x_{s l}^{i}\right)^{\frac{1}{L}} .
\end{aligned}
$$

Therefore,

$$
U_{s, x}(Q)=\left\{u_{s} \in \mathbb{R}^{Q} \mid \sum_{i \in Q} \exp \left(u_{s}^{i}\right) \leq \beta_{s, x}(Q)\right\},
$$

and

$$
\begin{aligned}
& C\left(M, U_{s, x}\right)=\left\{\quad u_{s} \in \mathbb{R}^{M} \mid\right. \\
& \sum_{i \in M} \exp \left(u_{s}^{i}\right)=\beta_{s, x}(M) \\
& \left.\sum_{i \in Q} \exp \left(u_{s}^{i}\right) \quad \geq \beta_{s, x}(Q), Q \subset M\right\} .
\end{aligned}
$$

If we define $\beta(\emptyset)=0$, then (11) can be rewritten as

$$
\begin{aligned}
& C\left(M, U_{s, x}\right)=\left\{\quad u_{s} \in \mathbb{R}^{M} \mid\right. \\
& \sum_{i \in M} \exp \left(u_{s}^{i}\right)=\beta_{s, x}(M) \\
& \left.\sum_{i \in Q} \exp \left(u_{s}^{i}\right) \leq \beta_{s, x}(M)-\beta_{s, x}(M / Q), Q \subset M\right\} \text {. }
\end{aligned}
$$


Therefore,

$$
\begin{array}{ll}
C\left(M, U_{s, x}\right) & -\mathbb{R}_{+}^{M}= \\
\left\{u_{s} \in \mathbb{R}^{M}\right. & \left.\mid \sum_{i \in Q} \exp \left(u_{s}^{i}\right) \leq \beta_{s, x}(M)-\beta_{s, x}(M / Q), Q \subseteq M\right\} .
\end{array}
$$

\section{$6 \quad$ Examples}

The purpose of the two examples reported below is to demonstrate some limitations of the concept of the weak sequential core. In Example 1 an economy with three agents and no assets is shown to have an empty weak sequential core. In contrast, the economy in Example 2, also with three agents and no assets, has a non-empty weak sequential core. It is shown, however, that the weak sequential core becomes empty, when an appropriately specified asset is introduced into the economy.

Example 1 Consider an economy in which $S=2, L=3$ and there are no assets. No restrictions are imposed on the probabilities of states, apart from $\rho_{1}, \rho_{2}>0$. There are three agents $N=\{a, b, c\}$ with identical consumption sets and identical state-independent Bernoulli utility functions given by (7) and (8). The initial endowments in the two states of nature are given by

$$
\begin{aligned}
& \omega_{1}=\left(\omega_{1}^{a}, \omega_{1}^{b}, \omega_{1}^{c}\right)=\left(\begin{array}{lll}
1 & \epsilon & 1 \\
\epsilon & 1 & 1 \\
\epsilon & 1 & 1
\end{array}\right) \\
& \omega_{2}=\left(\omega_{2}^{a}, \omega_{2}^{b}, \omega_{2}^{c}\right)=\left(\begin{array}{lll}
\epsilon & 1 & 1 \\
1 & \epsilon & 1 \\
1 & \epsilon & 1
\end{array}\right)
\end{aligned}
$$

It turns out that the weak sequential core of this economy is empty whenever $0<\epsilon \leq 0.021$. Before we come to a formal proof let us give some intuition for the emptiness result.

In Figure 1 the set $C\left(\{a, b\}, U_{s, \omega}\right)-\mathbb{R}^{\{a, b\}}$ and the projection of the set $C\left(N, U_{s, \omega}\right)-\mathbb{R}^{N}$ onto the space of utilities for agents $a$ and $b$ in the state $s=1$ are depicted. Figure 2 depicts the same objects for the state $s=2$. Figure 3 depicts the set $V(\{a, b\})$ and the projection of the set $V(\{a, b, c\})$ onto $\mathbb{R}^{\{a, b\}}$.

Observe that the vectors of the aggregate endowments $\omega_{s}^{a}+\omega_{s}^{b}$ owned by agents $a$ and $b$ are equal across the states. Since the elementary utility functions of both agents $a$ and $b$ are state-independent, 
the feasibility sets $U_{1, \omega}(\{a, b\})$ and $U_{2, \omega}(\{a, b\})$ in the two states of the world coincide. As $\epsilon$ gets smaller, the conditions of individual rationality for agents $a$ and $b$ become negligible. Therefore, the cores $C\left(\{a, b\}, U_{s, \omega}\right)$ are "almost" (up to the vanishing conditions of individual rationality) identical across the states of nature. This indicates the possibility of credible agreements within a coalition $\{a, b\}$ upon the state-independent (certain) allocation of utilities.

Due to the acute choice of values for the initial endowments, the core of the game $\left(\{a, b, c\}, U_{s, \omega}\right)$, when projected into the space $\mathbb{R}^{\{a, b\}}$, is a subset of $C\left(\{a, b\}, U_{s, \omega}\right)$. Moreover, the sets $C\left(\{a, b, c\}, U_{1, \omega}\right)$ and $C\left(\{a, b, c\}, U_{2, \omega}\right)$ are disjoint: the grand coalition is not able to agree upon any allocation of commodities under which agents $a$ and $b$ would enjoy state-independent utilities.

The risk aversion of the agents $a$ and $b$ reflected here by the strict convexity of the sets $U_{1, \omega}(\{a, b\})$ and $U_{2, \omega}(\{a, b\})$ implies that the coalition $\{a, b\}$ can improve upon any expected utility profile in the set $V(N)$. That is, $\left(v^{a}, v^{b}\right) \in \operatorname{int} V(\{a, b\})$ for all $v \in V(\{a, b, c\})$. Below we give a formal proof of this result.

Proof. Let $(N, V)$ be the NTU-game as defined in Section 5. We show that $C(N, V)=\oslash$, whenever $\epsilon$ is close enough to zero to satisfy the following inequalities:

$$
\begin{aligned}
& 0<\epsilon<1, \\
& 2+\epsilon-(4(1+\epsilon))^{\frac{1}{3}}<(4(1+\epsilon))^{\frac{1}{3}}-1, \\
& 2+\epsilon-\left(2(1+\epsilon)^{2}\right)^{\frac{1}{3}}<1+\epsilon-\epsilon^{\frac{1}{3}} .
\end{aligned}
$$

Notice that each $\epsilon$ in the interval $(0 ; 0.021]$ satisfies the inequality constraints.

Table 1 reports the values for $\beta_{s, \omega}(Q)$ as defined in (9). Using these values and the formula (13) in Section 5, it is easy to see that for coalition $\{a, b\}$

$$
\begin{aligned}
C\left(\{a, b\}, U_{1, \omega}\right)-\mathbb{R}_{+}^{\{a, b\}}= & \\
\left\{u_{1} \in \mathbb{R}^{\{a, b\}} \mid \exp \left(u_{1}^{a}\right)+\exp \left(u_{1}^{b}\right)\right. & \leq 1+\epsilon \\
\exp \left(u_{1}^{a}\right) & \leq 1+\epsilon-\epsilon^{\frac{1}{3}} \\
\exp \left(u_{1}^{b}\right) & \left.\leq 1+\epsilon-\epsilon^{\frac{2}{3}}\right\},
\end{aligned}
$$


Table 1: The values for $\beta_{s, \omega}(Q)$

\begin{tabular}{lll}
\hline & $s=1$ & $s=2$ \\
$Q=\{a\}$ & $\epsilon^{\frac{2}{3}}$ & $\epsilon^{\frac{1}{3}}$ \\
$Q=\{b\}$ & $\epsilon^{\frac{1}{3}}$ & $\epsilon^{\frac{2}{3}}$ \\
$Q=\{c\}$ & 1 & 1 \\
$Q=\{a, b\}$ & $1+\epsilon$ & $1+\epsilon$ \\
$Q=\{a, c\}$ & $\left(2(1+\epsilon)^{2}\right)^{\frac{1}{3}}$ & $(4(1+\epsilon))^{\frac{1}{3}}$ \\
$Q=\{b, c\}$ & $(4(1+\epsilon))^{\frac{1}{3}}$ & $\left(2(1+\epsilon)^{2}\right)^{\frac{1}{3}}$ \\
$Q=\{a, b, c\}$ & $2+\epsilon$ & $2+\epsilon$ \\
\hline
\end{tabular}

and

$$
\begin{aligned}
& C\left(\{a, b\}, U_{2, \omega}\right)-\mathbb{R}_{+}^{\{a, b\}}= \\
& \left\{u_{2} \in \mathbb{R}^{\{a, b\}} \mid \exp \left(u_{2}^{a}\right)+\exp \left(u_{2}^{b}\right) \leq 1+\epsilon\right. \\
& \exp \left(u_{2}^{a}\right) \leq 1+\epsilon-\epsilon^{\frac{2}{3}} \\
& \left.\exp \left(u_{2}^{b}\right) \leq 1+\epsilon-\epsilon^{\frac{1}{3}}\right\} .
\end{aligned}
$$

For the grand coalition

$$
\begin{aligned}
& C\left(N, U_{1, \omega}\right)= \\
& \left\{u_{1} \in \mathbb{R}^{N} \mid \exp \left(u_{1}^{a}\right)+\exp \left(u_{1}^{b}\right)=1+\epsilon, \quad u_{1}^{c}=0\right. \\
& \exp \left(u_{1}^{a}\right) \quad \leq 2+\epsilon-(4(1+\epsilon))^{\frac{1}{3}} \\
& \left.\left.\exp \left(u_{1}^{b}\right) \leq 2+\epsilon-\left(2(1+\epsilon)^{2}\right)^{\frac{1}{3}}\right\} 14\right)
\end{aligned}
$$

and

$$
\begin{aligned}
& C\left(N, U_{2, \omega}\right)= \\
& \left\{u_{2} \in \mathbb{R}^{N} \mid \exp \left(u_{2}^{a}\right)+\exp \left(u_{2}^{b}\right)=1+\epsilon, \quad u_{2}^{c}=0\right. \\
& \exp \left(u_{2}^{a}\right) \quad \leq 2+\epsilon-\left(2(1+\epsilon)^{2}\right)^{\frac{1}{3}} \\
& \left.\exp \left(u_{2}^{b}\right) \leq 2+\epsilon-(4(1+\epsilon))^{\frac{1}{3}}\right\}(15)
\end{aligned}
$$


We proceed by demonstrating that the projection of $V(N)$ onto the space of utilities for agents $a$ and $b$ lies entirely in the interior of the set $V(\{a, b\})$.

Step 1. $u_{s} \in C\left(N, U_{s, \omega}\right) \Rightarrow u_{1}^{a}<u_{2}^{a}, u_{1}^{b}>u_{2}^{b}$

Take any $u_{s} \in C\left(N, U_{s, \omega}\right), s=1,2$. One can see from (14) and (15) that the following inequalities hold:

$$
\begin{aligned}
& \exp \left(u_{1}^{a}\right) \leq 2+\epsilon-(4(1+\epsilon))^{\frac{1}{3}} \\
& \exp \left(u_{2}^{a}\right) \geq(4(1+\epsilon))^{\frac{1}{3}}-1 .
\end{aligned}
$$

The inequality constraints imposed upon $\epsilon$ therefore guarantee that $\exp \left(u_{1}^{a}\right)<\exp \left(u_{2}^{a}\right)$. Since

$$
\exp \left(u_{s}^{a}\right)+\exp \left(u_{s}^{b}\right)=1+\epsilon, \quad s=1,2,
$$

it follows that $\exp \left(u_{1}^{b}\right)>\exp \left(u_{2}^{b}\right)$.

Step 2.

$$
v \in V(N) \Rightarrow\left(v^{a}, v^{b}\right) \in \operatorname{int}\left(C\left(\{a, b\}, U_{s, \omega}\right)-\mathbb{R}_{+}^{\{a, b\}}\right), s=1,2 .
$$

It suffices to show that for any $v \in V(N)$ the following inequalities hold true:

$$
\begin{aligned}
\exp \left(v^{a}\right)+\exp \left(v^{b}\right) & <1+\epsilon \\
\exp \left(v^{i}\right) & <1+\epsilon-\epsilon^{\frac{1}{3}}, \quad i=a, b .
\end{aligned}
$$

Choose $u_{s} \in C\left(N, U_{s, \omega}\right)$ so that $v \leq \rho_{1} u_{1}+\rho_{2} u_{2}$. The fact that $u_{1}^{a} \neq u_{2}^{a}$, the strict convexity of the exponential function, and the equations (16) yield the inequalities

$$
\begin{array}{r}
\exp \left(v^{a}\right)+\exp \left(v^{b}\right) \leq \\
\leq \exp \left(\rho_{1} u_{1}^{a}+\rho_{2} u_{2}^{a}\right)+\exp \left(\rho_{1} u_{1}^{b}+\rho_{2} u_{2}^{b}\right)< \\
<\rho_{1} \exp \left(u_{1}^{a}\right)+\rho_{2} \exp \left(u_{2}^{a}\right)+\rho_{1} \exp \left(u_{1}^{b}\right)+\rho_{2} \exp \left(u_{2}^{b}\right)= \\
=\rho_{1}\left(\exp \left(u_{1}^{a}\right)+\exp \left(u_{1}^{b}\right)\right)+\rho_{2}\left(\exp \left(u_{2}^{a}\right)+\exp \left(u_{2}^{b}\right)\right)= \\
=1+\epsilon .
\end{array}
$$

It follows from step 1 that

$$
\begin{aligned}
& \exp \left(v^{a}\right)<\exp \left(u_{2}^{a}\right), \quad \text { and } \\
& \exp \left(v^{b}\right)<\exp \left(u_{1}^{b}\right) .
\end{aligned}
$$


As is evident from (14) and (15), both $\exp \left(u_{2}^{a}\right)$ and $\exp \left(u_{1}^{b}\right)$ are less than or equal to $\left(2+\epsilon-\left(2(1+\epsilon)^{2}\right)^{\frac{1}{3}}\right)$, which, under the inequality constraints imposed upon $\epsilon$, is less than $\left(1+\epsilon-\epsilon^{1 / 3}\right)$.

It follows at once from step 2 that $\left(v^{a}, v^{b}\right) \in \operatorname{int} V(\{a, b\})$ for all $v \in V(N)$.

Example 2 Consider an economy in which $S=2, L=2$, and $\rho_{1}=\rho_{2}=0.5$. There are three agents $N=\{a, b, c\}$ with identical consumption sets and identical state-independent Bernoulli utility functions given by (7) and (8). The initial endowments in the two states of nature are given by

$$
\begin{aligned}
& \omega_{1}=\left(\omega_{1}^{a}, \omega_{1}^{b}, \omega_{1}^{c}\right)=\left(\begin{array}{lll}
1 & \epsilon & 1 \\
1 & \epsilon & 1
\end{array}\right) \\
& \omega_{2}=\left(\omega_{2}^{a}, \omega_{2}^{b}, \omega_{2}^{c}\right)=\left(\begin{array}{lll}
1 & 1 & \epsilon \\
1 & \epsilon & 1
\end{array}\right)
\end{aligned}
$$

Suppose that there are no assets in the economy. Then the weak sequential core is given by

$$
W S C(\mathcal{E})=\left\{\omega_{1}\right\} \times C\left(\mathcal{E}_{2}(\omega, N)\right) .
$$

Proof. Note that for any coalition $M \subseteq N$ the core of the economy $\mathcal{E}_{1}(\omega, M)$ contains a unique allocation: that of the initial endowments. Formally,

$$
C\left(\mathcal{E}_{1}(\omega, M)\right)=\left\{\left(\omega_{1}^{i}\right)_{i \in M}\right\} .
$$

With this observation at hand, the definition of the weak sequential core for the economy in question reduces to the following:

An allocation $\bar{y}$ is an element of the weak sequential core if and only if

1. $\bar{y}_{1}=\omega_{1}$ and $\bar{y}_{2} \in C\left(\mathcal{E}_{2}(\omega, N)\right)$

2. there do not exist $M \subseteq N$ and $y \in \prod_{i \in M} X^{i}$ such that

$y_{1}=\left\{\left(\omega_{1}^{i}\right)_{i \in M}\right\}$

$y_{2} \in C\left(\mathcal{E}_{2}(\omega, M)\right)$

$v^{i}\left(y^{i}\right)>v^{i}\left(\bar{y}^{i}\right) \quad \forall i \in M$. 
In this reduced definition the equation $v^{i}\left(y^{i}\right)>v^{i}\left(\bar{y}^{i}\right)$ may be replaced by $u_{2}^{i}\left(y_{2}^{i}\right)>u_{2}^{i}\left(\bar{y}_{2}^{i}\right)$. Therefore, the set of allocations $\left(\bar{y}_{1}, \bar{y}_{2}\right)$ satisfying requirements 1 and 2 is $\left\{\omega_{1}\right\} \times C\left(\mathcal{E}_{2}(\omega, N)\right)$.

Suppose, instead, that there is a single asset in the economy $(J=$ 1 ), of which the payoffs in the two states are given by

$$
A_{1}=\left(\begin{array}{l}
1 \\
0
\end{array}\right), A_{2}=\left(\begin{array}{l}
0 \\
0
\end{array}\right)
$$

i.e. the asset is a contract for the delivery of commodity 1 in state 1 . Then the weak sequential core is empty whenever $0<\epsilon \leq 0.038$.

Proof. Let $(N, V)$ be the NTU-game defined as in the Section 5. We show that $C(N, V)=\oslash$, whenever $\epsilon$ is close enough to zero to satisfy the following inequalities:

$$
\begin{aligned}
& 0<\epsilon<1 \\
& \frac{1}{1-\epsilon^{\frac{1}{2}}}+\epsilon^{\frac{1}{2}}<(2+2 \epsilon)^{\frac{1}{2}} \\
& 1+\left(2+\epsilon-(2+2 \epsilon)^{\frac{1}{2}}\right)^{2}<(2+2 \epsilon)^{\frac{1}{2}} .
\end{aligned}
$$

Observe that each $\epsilon$ in the interval $(0,0.038]$ satisfies the inequality constraints.

The proof is split in two parts. First we construct some of the feasible expected utility tuples - subsets of or isolated points in $V(Q)$ - for various sub-coalitions $Q$ of $N$. Then we demonstrate that the conditions

$$
\left\{\begin{array}{l}
\bar{v} \in V(N) \\
\bar{v}^{Q} \notin \operatorname{int} V(Q), \forall Q \subseteq N
\end{array}\right.
$$

are incompatible.

\section{Part 1.}

Notice first that for any coalition $Q \subseteq N$ and any $A$-feasible allocation $x \in \mathcal{A}(Q)$ it holds that $x_{2}=\left(\omega_{2}^{i}\right)_{i \in Q}$. In what follows $x$ is defined by its component $x_{1}$, corresponding to the first state of the world.

For the single-element coalitions $\{a\}$ and $\{c\}$

$$
V(\{a\})=(-\infty ; 0] \text {, and }
$$




$$
V(\{c\})=\left(-\infty ; \ln \epsilon^{\frac{1}{4}}\right] .
$$

For the coalition $\{b, c\}$ it holds that

$$
\begin{aligned}
& C\left(\{b, c\}, U_{2, \omega}\right)-\mathbb{R}_{+}^{\{b, c\}}= \\
& \left\{u_{2} \in \mathbb{R}^{\{b, c\}} \mid \exp \left(u_{2}^{b}\right)+\exp \left(u_{2}^{c}\right) \leq 1+\epsilon\right. \\
& \exp \left(u_{2}^{b}\right) \quad \leq 1+\epsilon-\epsilon^{\frac{1}{2}} \\
& \exp \left(u_{2}^{c}\right) \leq 1+\epsilon-\epsilon\left(\frac{1}{22}\right) .
\end{aligned}
$$

Let the allocation $x \in \mathcal{A}(\{b, c\})$ be defined by

$$
x_{1}=\left(x_{1}^{b}, x_{1}^{c}\right)=\left(\begin{array}{ll}
1 & \epsilon \\
\epsilon & 1
\end{array}\right) .
$$

Since $x_{1}=x_{2}=\left(\omega_{2}^{b}, \omega_{2}^{c}\right)$, the sets $\left(C\left(\{b, c\}, U_{s, x}\right)-\mathbb{R}_{+}^{\{b, c\}}\right), s=1,2$, coincide with the set defined by (20). Therefore $V_{x}(\{b, c\})$ also equals (20):

$$
\begin{aligned}
V_{x}(\{b, c\})=\left\{v \in \mathbb{R}^{\{b, c\}} \mid \exp \left(v^{b}\right)+\exp \left(v^{c}\right)\right. & \leq 1+\epsilon \\
\exp \left(v^{b}\right) & \leq 1+\epsilon-\epsilon^{\frac{1}{2}} \\
\exp \left(v^{c}\right) & \left.\left.\leq 1+\epsilon-\epsilon^{\frac{1}{2}}\right\}^{2} 1\right)
\end{aligned}
$$

Consider next coalition $\{a, b\}$. Let the allocation $x \in \mathcal{A}(\{a, b\})$ be defined by

$$
x_{1}=\left(x_{1}^{a}, x_{1}^{b}\right)=\left(\begin{array}{cc}
\epsilon & 1 \\
1 & \epsilon
\end{array}\right) .
$$

Then

$$
\begin{aligned}
C\left(\{a, b\}, U_{1, x}\right)-\mathbb{R}_{+}^{\{a, b\}}= & \\
\left\{u_{1} \in \mathbb{R}^{\{a, b\}} \mid \exp \left(u_{1}^{a}\right)+\exp \left(u_{1}^{b}\right)\right. & \leq 1+\epsilon \\
\exp \left(u_{1}^{a}\right) \quad & \leq 1+\epsilon-\epsilon^{\frac{1}{2}} \\
\exp \left(u_{1}^{b}\right) & \left.\leq 1+\epsilon-\epsilon^{\frac{1}{2}}\right\},
\end{aligned}
$$


and

$$
\begin{aligned}
& C\left(\{a, b\}, U_{2, x}\right)-\mathbb{R}_{+}^{\{a, b\}}= \\
& \left\{u_{2} \in \mathbb{R}^{\{a, b\}} \mid \exp \left(u_{2}^{a}\right)+\exp \left(u_{2}^{b}\right) \leq(2(1+\epsilon))^{\frac{1}{2}}\right. \\
& \exp \left(u_{2}^{a}\right) \quad \leq(2(1+\epsilon))^{\frac{1}{2}}-\epsilon^{\frac{1}{2}} \\
& \left.\exp \left(u_{2}^{b}\right) \leq(2(1+\epsilon))^{\frac{1}{2}}-1\right\} \text {. }
\end{aligned}
$$

Define $u_{s} \in \mathbb{R}^{\{a, b\}}$ as follows:

$$
\begin{aligned}
& \left(u_{1}^{a}, u_{1}^{b}\right)=\left(\ln \left(1-\epsilon^{\frac{1}{2}}\right), \ln \left(\epsilon^{\frac{1}{2}}\right)\right) \\
& \left(u_{2}^{a}, u_{2}^{b}\right)=\left(-\ln \left(1-\epsilon^{\frac{1}{2}}\right), \ln \left(\epsilon^{\frac{1}{2}}\right)\right) .
\end{aligned}
$$

Under the inequality constraints imposed upon $\epsilon$, it holds that

$$
u_{s} \in \operatorname{int}\left(C\left(\{a, b\}, U_{s, x}\right)-\mathbb{R}_{+}^{\{a, b\}}\right)
$$

for $s=1,2$. Therefore,

$$
0.5 u_{1}+0.5 u_{2}=\left(0, \ln \epsilon^{\frac{1}{2}}\right) \in \operatorname{int} V_{x}(\{a, b\}) .
$$

For the coalition $\{a, c\}$ it holds that

$$
\begin{aligned}
& C\left(\{a, c\}, U_{1, \omega}\right)=\{(0,0)\} \\
& C\left(\{a, c\}, U_{2, \omega}\right)-\mathbb{R}_{+}^{\{a, c\}}= \\
& \left\{u_{2} \in \mathbb{R}^{\{a, c\}} \mid \begin{array}{ll}
\exp \left(u_{2}^{a}\right)+\exp \left(u_{2}^{c}\right) & \leq(2(1+\epsilon))^{\frac{1}{2}} \\
& \exp \left(u_{2}^{a}\right) \\
& \leq(2(1+\epsilon))^{\frac{1}{2}}-\epsilon^{\frac{1}{2}} \\
\exp \left(u_{2}^{c}\right) & \left.\leq(2(1+\epsilon))^{\frac{1}{2}}-1\right\} .
\end{array}\right.
\end{aligned}
$$

Define $u_{s} \in \mathbb{R}^{\{a, c\}}$ as follows:

$$
\begin{aligned}
& \left(u_{1}^{a}, u_{1}^{c}\right)=(0,0) \\
& \left(u_{2}^{a}, u_{2}^{c}\right)=\left(0,2 \ln \left(2+\epsilon-(2+2 \epsilon)^{\frac{1}{2}}\right)\right) .
\end{aligned}
$$

Under the inequality constraints imposed upon $\epsilon$ it holds that

$$
u_{2} \in \operatorname{int}\left(C\left(\{a, c\}, U_{2, \omega}\right)-\mathbb{R}_{+}^{\{a, c\}}\right) .
$$


Hence,

$$
0.5 u_{1}+0.5 u_{2}=\left(0, \ln \left(2+\epsilon-(2+2 \epsilon)^{\frac{1}{2}}\right)\right) \in \operatorname{int} V(\{a, c\}) .
$$

Finally, consider the grand coalition. It holds that

$$
\begin{aligned}
& C\left(N, U_{2, \omega}\right)-\mathbb{R}_{+}^{N}= \\
& \left\{u_{2} \in \mathbb{R}^{N} \mid \quad u_{2}^{a} \quad \leq 0\right. \\
& \exp \left(u_{2}^{b}\right)+\exp \left(u_{2}^{c}\right) \leq 1+\epsilon \\
& \exp \left(u_{2}^{b}\right) \quad \leq 2+\epsilon-(2+2 \epsilon)^{\frac{1}{2}} \\
& \exp \left(u_{2}^{c}\right) \leq 2+\epsilon-(2+2 \epsilon)\left(\frac{1}{2}\right)
\end{aligned}
$$

For any $\bar{x} \in \mathcal{A}(N)$ and for $s=1,2$

$$
U_{s, \bar{x}}(N)=\left\{u_{s} \in \mathbb{R}^{N} \mid \exp \left(u_{s}^{a}\right)+\exp \left(u_{s}^{b}\right)+\exp \left(u_{s}^{c}\right) \leq 2+\epsilon\right\} .
$$

It follows from (25) that

$$
V(N) \subseteq\left\{v \in \mathbb{R}^{N} \mid \exp \left(v^{a}\right)+\exp \left(v^{b}\right)+\exp \left(v^{c}\right) \leq 2+\epsilon\right\} .
$$

Part 2.

Let $\bar{v}$ be an element of $C(N, V)$. Let $\bar{x} \in \mathcal{A}(N)$ and $\bar{u}_{s} \in \mathbb{R}^{N}, s=1,2$, be such that

$$
\begin{aligned}
\bar{u}_{s} & \in\left(C\left(N, U_{s, \bar{x}}\right)-\mathbb{R}_{+}^{N}\right), s=1,2 \\
\bar{v} & =0.5 \bar{u}_{1}+0.5 \bar{u}_{2} .
\end{aligned}
$$

Step 1. $\quad \exp \left(\bar{u}_{1}^{b}\right)+\exp \left(\bar{u}_{1}^{c}\right) \geq 1+\epsilon$

We show that if $\exp \left(\bar{u}_{1}^{b}\right)+\exp \left(\bar{u}_{1}^{c}\right)<1+\epsilon$, then coalition $\{b, c\}$ has a credible deviation from $\bar{v}$. Choose a utility profile $\hat{u}_{1} \in \mathbb{R}^{\{b, c\}}$ such that

$$
\begin{array}{r}
\exp \left(\hat{u}_{1}^{b}\right)+\exp \left(\hat{u}_{1}^{c}\right)=1+\epsilon \\
\left(\hat{u}_{1}^{b}, \hat{u}_{1}^{c}\right)>>\left(\bar{u}_{1}^{b}, \bar{u}_{1}^{c}\right) .
\end{array}
$$


Let the allocation $x \in \mathcal{A}(\{b, c\})$ be defined by

$$
\begin{aligned}
x_{1} & =\left(x^{b}, x^{c}\right)=\left(\begin{array}{cc}
\epsilon+\theta & 1-\theta \\
\epsilon & 1
\end{array}\right), \text { where } \\
\theta & = \begin{cases}\frac{\exp \left(2 \hat{u}_{1}^{b}\right)}{\epsilon}-\epsilon & \text { if } \hat{u}_{1}^{c} \geq 0 \\
1-\exp \left(2 \hat{u}_{1}^{c}\right), & \text { otherwise. }\end{cases}
\end{aligned}
$$

We leave it to the reader to show that $x_{1,1}^{i}>0, i=b, c$, and that

$$
\hat{u}_{1}^{i} \geq u_{1}^{i}\left(x_{1}^{i}\right), \quad i \in\{b, c\} .
$$

It follows from (28)-(29) that $\hat{u}_{1} \in C\left(\{b, c\}, U_{1, x}\right)$, and therefore

$$
\left(\bar{u}_{1}^{b}, \bar{u}_{1}^{c}\right) \in \operatorname{int}\left(C\left(\{b, c\}, U_{1, x}\right)-\mathbb{R}_{+}^{\{b, c\}}\right) .
$$

Comparing equations (20) and (24) we notice that the inclusion

$$
\bar{u} \in\left(C\left(N, U_{2, \omega}\right)-\mathbb{R}_{+}^{N}\right)
$$

implies the inclusion

$$
\left(\bar{u}_{2}^{b}, \bar{u}_{2}^{c}\right) \in\left(C\left(\{b, c\}, U_{2, \omega}\right)-\mathbb{R}_{+}^{\{b, c\}}\right) .
$$

Finally, (30) and (31) lead to the conclusion that $\left(\bar{v}^{b}, \bar{v}^{c}\right) \in \operatorname{int} V_{x}(\{b, c\})$.

Step 2.

$$
\begin{aligned}
& \bar{u}_{s}^{a}=\bar{v}^{a}=0, s=1,2, \\
& \exp \left(\bar{u}_{s}^{b}\right)+\exp \left(\bar{u}_{s}^{c}\right) \leq 1+\epsilon, s=1,2 .
\end{aligned}
$$

It follows from (24) that $\bar{u}_{2}^{a} \leq 0$. The fact that $\bar{u}_{1} \in U_{1, \bar{x}}(N)$ (see (25)) together with equation (27) imply that $\bar{u}_{1}^{a} \leq 0$. This gives $\bar{v}^{a} \leq 0$. On the other hand, the requirement that $\bar{v}^{a}$ be outside intV $(\{a\})$ implies $\bar{v}^{a} \geq 0$ (see (18)). Thus we proved (32). Equations (33) are now implied by (32) and the fact that $\bar{u}_{s} \in U_{s, \bar{x}}(N)$ (see (25)).

Step 3. $\quad \exp \left(\bar{v}^{b}\right)+\exp \left(\bar{v}^{c}\right)=1+\epsilon$. 
From (26) and the fact that $\bar{v}^{a}=0$ it follows that

$$
\exp \left(\bar{v}^{b}\right)+\exp \left(\bar{v}^{c}\right) \leq 1+\epsilon .
$$

The requirement that the utility profile $\left(\bar{v}^{b}, \bar{v}^{c}\right)$ be outside the interior of the set defined by (21) implies that at least one of the following inequalities are satisfied:

$$
\begin{aligned}
\exp \left(\bar{v}^{b}\right)+\exp \left(\bar{v}^{c}\right) & \geq 1+\epsilon \\
\exp \left(\bar{v}^{b}\right) & \geq 1+\epsilon-\epsilon^{\frac{1}{2}} \\
\exp \left(\bar{v}^{c}\right) & \geq 1+\epsilon-\epsilon^{\frac{1}{2}} .
\end{aligned}
$$

If inequality (37) were satisfied, then, together with (35), it would give $\exp \left(\bar{v}^{c}\right) \leq \epsilon^{\frac{1}{2}}$. Then $\bar{v}^{c}$ would lie in the interior of the set $V(\{c\})$ (see (19)), which cannot be the case.

If inequality (38) were satisfied, then, together with (35), it would give $\exp \left(\bar{v}^{b}\right) \leq \epsilon^{\frac{1}{2}}$. Hence, $\left(\bar{v}^{a}, \bar{v}^{b}\right) \leq\left(0, \ln \epsilon^{\frac{1}{2}}\right)$. Inclusion (22) would then imply that the utility tuple $\left(\bar{v}^{a}, \bar{v}^{b}\right)$ lie in the interior of the set $V(\{a, b\})$, which cannot be the case.

Since both (37) and (38) are violated, (36) must be satisfied. Equations (35) and (36) produce (34).

Step 4. $\quad\left(\bar{u}_{1}^{b}, \bar{u}_{1}^{c}\right)=\left(\bar{u}_{2}^{b}, \bar{u}_{2}^{c}\right)$.

If this equality was violated, then, due to the strict convexity of the exponential function and inequalities (33)

$$
\begin{aligned}
\exp \left(\bar{v}^{b}\right)+\exp \left(\bar{v}^{c}\right) & <0.5 \exp \left(\bar{u}_{1}^{b}\right)+0.5 \exp \left(\bar{u}_{2}^{b}\right)+0.5 \exp \left(\bar{u}_{1}^{c}\right)+0.5 \exp \left(\bar{u}_{2}^{c}\right) \\
& \leq 1+\epsilon .
\end{aligned}
$$

This last inequality, however, would contradict (34).

Step 5. $\quad\left(\bar{v}^{a}, \bar{v}^{c}\right) \in \operatorname{int} V(\{a, c\})$

Since $\bar{u}_{2} \in C\left(N, U_{2, \omega}\right)$, (see $\left.(24)\right) \bar{u}_{2}^{c}$ satisfies

$$
\exp \left(\bar{u}_{2}^{c}\right) \leq 2+\epsilon-(2+2 \epsilon)^{\frac{1}{2}}
$$


We know from the previous step that $\bar{u}_{2}^{c}=\bar{v}^{c}$. Hence,

$$
\left(\bar{v}^{a}, \bar{v}^{c}\right) \leq\left(0 ; \ln \left(2+\epsilon-(2+2 \epsilon)^{\frac{1}{2}}\right)\right) .
$$

The inclusion (23) implies that the utility tuple $\left(\bar{v}^{a}, \bar{v}^{c}\right)$ belongs to the interior of the set $V(\{a, c\})$, leading to a contradiction. 


\section{References}

[1] Becker, R.A. and S.K. Chakrabarti (1995): "The Recursive Core," Econometrica, 63, 401-423.

[2] Gale, D. (1978): "The Core of a Monetary Economy without Trust," Journal of Economic Theory, 19, 456-491.

[3] Geanakoplos, J.D., and H.M. Polemarchakis (1986), "Existence, Regularity, and Constrained Suboptimality of Competitive Allocations when the Asset Market is Incomplete," in W.P. Heller, R.M. Starr, and D.A. Starrett (eds.), Uncertainty, Information and Communication: Essays in Honor of K.J. Arrow, Vol. III, Cambridge University Press, Cambridge, pp. 65-96.

[4] Koutsougeras, L.C. (1998): "A Two-Stage Core With Applications to Asset Market and Differential Information Economies," Economic Theory, 11, 563-584.

[5] Kranich, L., A. Perea, and H. Peters (2001): "Dynamic Cooperation: Transferable Utility Games and Core Concepts," METEOR research memorandum.

[6] Predtetchinski, A., P.J.J. Herings, and H. Peters (2001): "The Strong Sequential Core for Two-period Economies," METEOR research memorandum.

[7] Ray, D. (1989): "Credible Coalitions and the Core," International Journal of Game Theory, 18, 185-197.

[8] Repullo, R. (1988): "The Core of an Economy with Transaction Costs," Review of Economic Studies, 55, 447-458. 


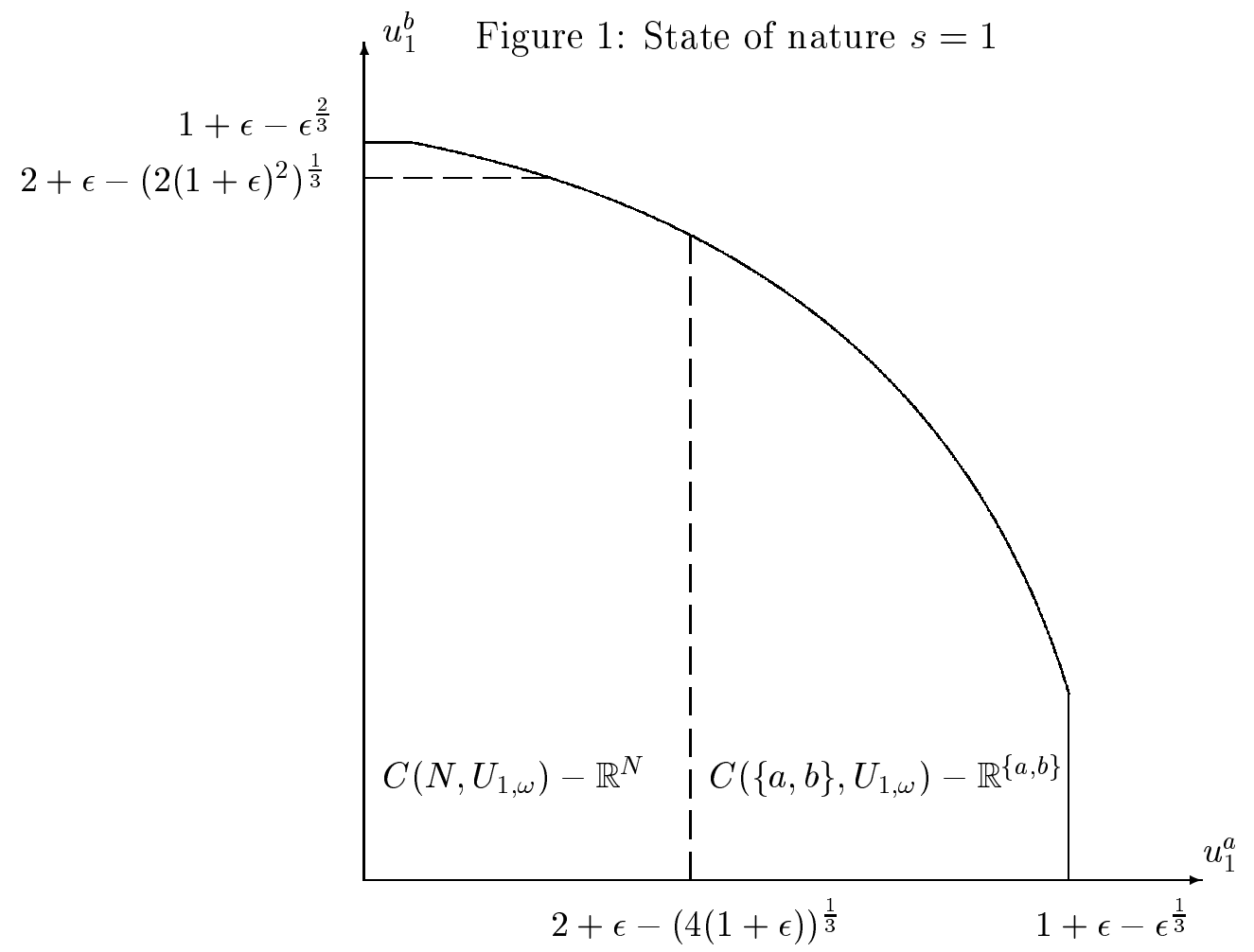




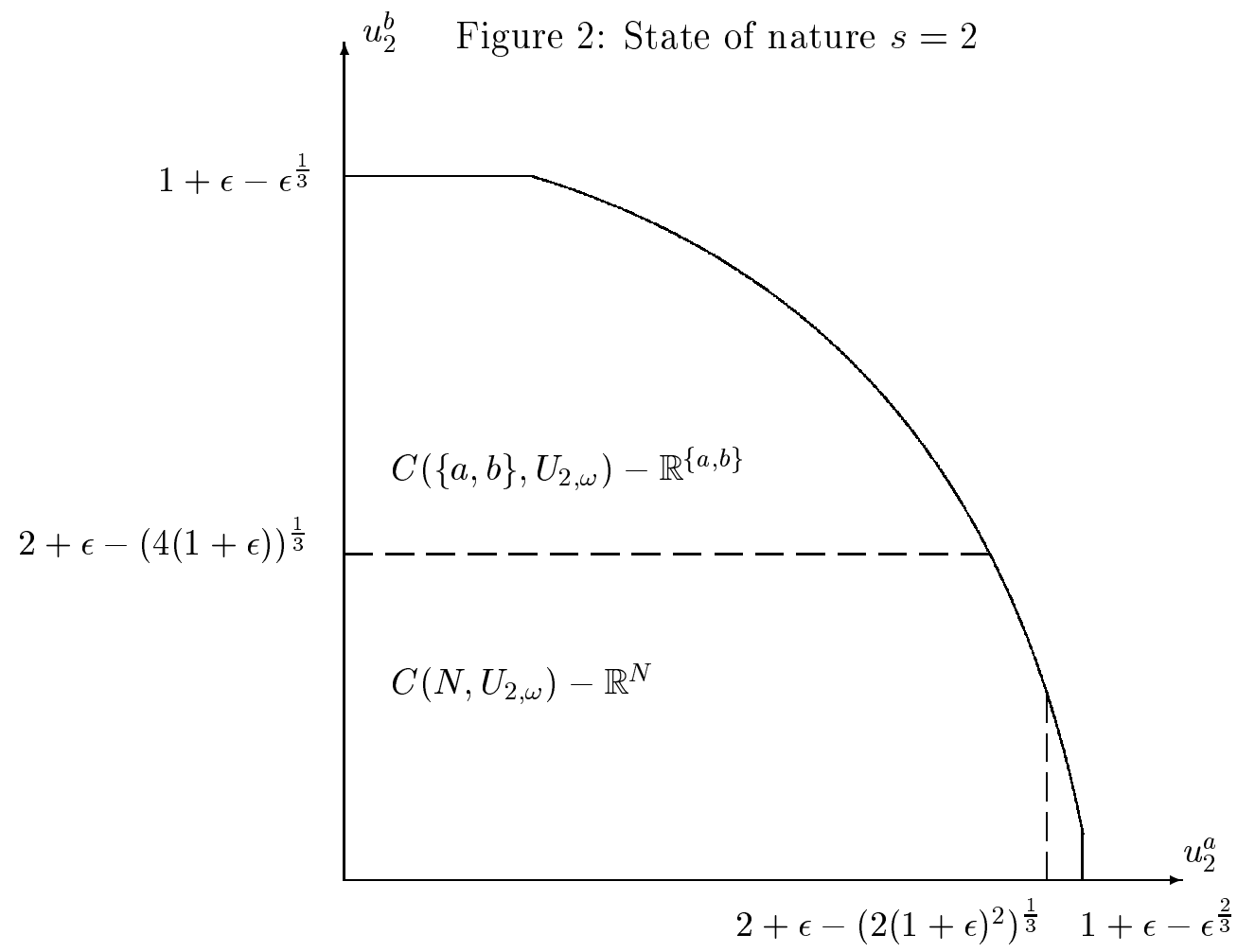




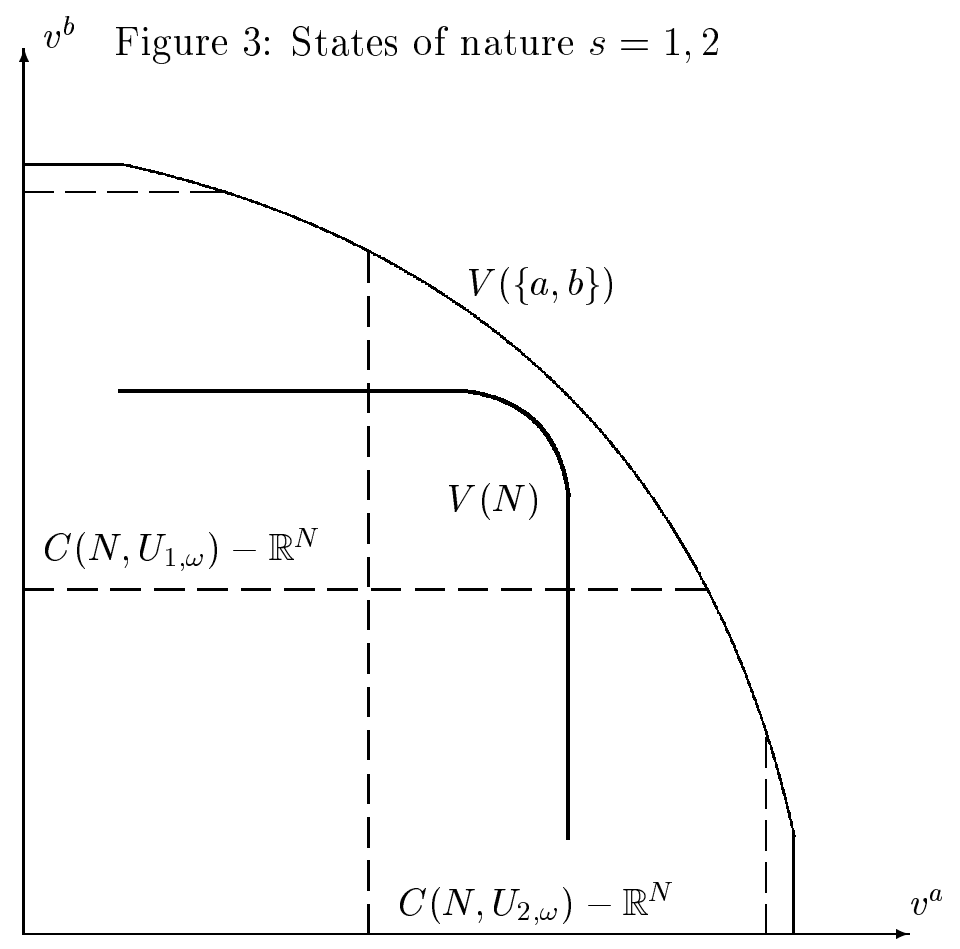

